\title{
The Floating of a Body on the Surface of a Liquid: A Synthesis
}

\author{
Giancarlo Cavazzini ${ }^{1}$ \\ ${ }^{1}$ Consiglio Nazionale delle Ricerche, Istituto di Geoscienze e Georisorse, Via G. Gradenigo, Padova, Italy \\ Correspondence: Giancarlo Cavazzini, Consiglio Nazionale delle Ricerche, Istituto di Geoscienze e Georisorse, \\ Via G. Gradenigo, 6, 35131, Padova, Italy. E-mail: giancarlo.cavazzini@igg.cnr.it
}

Received: March 4, 2021

doi:10.5539/apr.v13n1p33
Accepted: March 31, $2021 \quad$ Online Published: June 30, 2021

URL: http://dx.doi.org/10.5539/apr.v13n1p33

\begin{abstract}
The current physical interpretation of the phenomenon of floating of a body on the surface of a liquid - the so-called 'Archimedes' Principle' - is not correct. It is based on a assumption which is false, because it leads to contradict observed facts. A different physical interpretation is the correct interpretation, which is equivalent to the current one from the quantitative viewpoint, but significantly different from the physical viewpoint.

When a volume of matter - a body - is immersed in a fluid, it 'loses' its own weight, i.e., it is no longer home to the downward-directed mechanical action of which the volume is home when it is immersed in a vacuum. Therefore, a material volume immersed in a fluid does not host two mechanical actions - the 'weight' of the volume itself, directed downwards, and the 'Archimedes' force', directed upwards - but a single mechanical action. The intensity of this action is proportional to the volume of the body and to the difference in density between the matter which constitutes the body and the matter which constitutes the fluid. The direction of this action is related to the sign of the difference between the two values of density, reversing as the sign changes from positive to negative and vice-versa.

Since the intensity of this mechanical action is proportional to the difference in density between the matter which constitutes the body and the matter which constitutes the fluid, there is no mechanical action - i.e., the mechanical action ceases - if there is equilibrium in density between the matter of the body and the matter of the fluid. This indicates that the main cause of this mechanical action is a physical disequilibrium between the matter which constitutes the body and the matter which constitutes the fluid, i.e., a physical disequilibrium/relationship between matter which is in contact.
\end{abstract}

Keywords: Equilibrium of Floating, Concept of 'Weight'

Entia non sunt multiplicanda praeter necessitatem

William of Ockham

\section{Introduction}

Very frequently in everyday life, we see effects of the physical phenomenon called 'gravity' or 'gravitation', i.e., 'action due to gravity'. This physical process was investigated since the ancient ages, and its properties are still very discussed.

The main effects of this physical process are two. The first effect is the so-called 'weight' of a body, the phenomenon that makes bodies (volumes of matter) 'heavy'. The weight of a body is the result of our mechanical opposition to the tendency of the process to move the body towards the ground beneath our feet (we usually say 'downwards'). The weight of a body, i.e., the intensity of the mechanical action that we must exert on the body in the unit of time to prevent the process from moving the body downwards, all the other conditions being equal, is currently believed to be proportional to the 'mass' of the body, where 'mass' is defined as the product between the volume of the body and the 'density' of the matter which constitutes the body.

However, the value of density is measured by the weight of a standard volume of matter, the so-called 'unit of volume', i.e., it is measured by the intensity of the mechanical action that we must exert in the unit of time on the standard volume to prevent the process from moving it downwards. Since the intensity of our mechanical action 
changes depending on the matter in which the standard volume of the matter is immersed, the value of density must be referred to particular (standard) conditions. This means that the concept of 'weight', i.e., our definition/interpretation of 'weight', is limited by our hypotheses/decisions about this mechanical action.

If we do not exert any mechanical action to counteract the tendency of the process to move the body, leaving the body 'free', or if we exert an insufficient mechanical action, the process moves the body downwards. The motion of bodies is the second important commonly-observed effect of the gravitational process, and when we observe the motion of a body due to the action of gravity we usually say: 'The body is falling'. This sentence is ambiguous, however, because, despite the fact that the intensity of the weight of a body depends on some characteristics of the body, the body does not 'decide' to move itself downwards. Instead, it would be much more appropriate to say: 'The body is moved downwards by a process', i.e., a process is occurring, which is the result of some conditions, which causes the motion of the body, and the motion has an aim, a physical aim. This is useful to shift our conceptual perception about what we see happening.

Among the properties of the gravitational mechanical action, there is an important 'property', which is the topic of this study. The reason for this is that this 'property' is, in fact, an assumption, i.e., it is an hypothesis about the gravitational mechanical action itself. This hypothesis can be illustrated as follows:

The intensity and the direction of the gravitational mechanical action of which a body is home- i.e., the intensity and the direction of the 'weight' of a body - all the other conditions being equal, are unchanged whatever the matter in which the body is immersed.

In other words, all the other conditions being equal, the weight of a body is present, unchanged in intensity and direction, whatever the matter in which the body is immersed.

This hypothesis is underlying the interpretation of the phenomenon of floating of a body on the surface of a liquid, the so-called 'Archimedes' Principle' (Archimedes; Halliday et al., 2001; Leroy, 1985). This Principle, which is perhaps the most important law in hydrostatics, at the base of the concept of Isostasy in Geophysics (i.e., the relationship of floating of the Earth's crust on the Earth's mantle), is still debated today, because some aspects have not yet been exhaustively understood (Bierman \& Kincanon, 2003; Brown, 1999; Graf, 2004; Sears, 1963; Taibu, 2015; Taibu et al., 2015). Therefore, it is important to investigate the phenomenon of floating, in order to establish whether the hypothesis on the gravitational mechanical action is correct or not. This is the aim of this study, which is an argued synthesis of recent works (Cavazzini, 2018; 2019; 2020).

2. The Reason Why in the Case of the 'Gravitational' Mechanical Action It Is More Appropriate to Say That a Body 'Is Home to' the Mechanical Action and Not That the Body 'Is Subjected to' the Mechanical Action

When we refer to the mechanical action (currently also called 'force') we call 'weight' of a body, it is more appropriate to say that the body 'is home to' the mechanical action and not that the body 'is subjected to' the mechanical action, because the body, due to a physical process, becomes the home of the mechanical action that we perceive/observe. This is to emphasize the difference between this particular mechanical action and other mechanical actions, for example the mechanical action which we can exert on a body, an object resting on a table, to move the object. In this case, the mechanical action has nothing to do with the object, because the intensity of the mechanical action we can exert on the object can be any, small or large. Of course, to move the object we must produce a minimum mechanical effort, which will be different depending on whether the object is small or large, all the other conditions being equal. However, the intensity of the mechanical action is not strictly related to the object, i.e., to the properties of the object. Apart of the minimum mechanical effort which is necessary to start moving the object, the intensity of the mechanical action can be very different, assuming an infinite number of values.

This is not the case of the mechanical action we perceive if we hold a body in our hands. This action depends on the characteristics of the body, caeteris paribus. For example, it depends on the volume of the body, and it is the body, therefore, which is, to some extent, at the origin of the mechanical action itself.

If we interpret the gravitational mechanical action of which a body is home as caused by a 'gravitational field', this mechanical action is considered an action 'on' the body, i.e., external to the body, because the gravitational field is believed to exist independently of the existence of the body, as our muscular effort on the object resting on the table exists independently of the object.

As emphasized by Albert Einstein (Einstein, 1916), 'fields' are models, conceptual ways which are useful in tentatively interpreting actions which occur or seem to occur at a distance. This interpretative scenario, however, conflicts with the fact that the intensity of the mechanical action, caeteris paribus, is unquestionably related to 
the characteristics of the matter which constitutes the body.

\section{Equilibrium and Disequilibrium}

When a body is immersed in a vacuum, a process the mechanism of which is currently unknown to us causes the body to be home to a mechanical action whose direction is towards the ground beneath our feet. When a body, as in this case, is home to a net mechanical action, we say that the body is home to a 'mechanical disequilibrium'.

If we hold the body in our hands, the body, in its materiality, 'transfers' to our own body the mechanical action of which it is home. This action is perceived by us as the 'weight' or the 'heaviness' of the body, i.e., the mechanical perception which corresponds to the intensity of the mechanical action that we must exert in the unit of time to prevent the process from moving the body downwards.

We usually represent this mechanical action with an arrow: the direction of the arrow indicates the direction of the mechanical action, and the length of the arrow is proportional to the intensity of the action (Fig. 1 (a)),

Instead, when the same body is floating on the surface of a liquid (Fig. 1 (b)), no action is perceived, neither directed downwards nor directed upwards. If the body, when it is floating on the surface of the liquid, is still home to a downward-directed mechanical action, a mechanical action opposite in direction and equivalent in intensity must be simultaneously present, which 'cancels out' the former. We say that the body is in 'mechanical equilibrium', which means that mechanical actions are present, but the sum of these actions is such that the body is not moved (there is no net action).

On the other hand, if the body which floats on the surface of a liquid is not home to any downward-directed mechanical action, there is no action equivalent in intensity and opposite in direction. Therefore, first of all, we have to establish whether a body which floats on the surface of a liquid is home to a downward-directed mechanical action or not.

\section{A Body Which Floats on the Surface of a Liquid Is Home to a Downward-Directed Mechanical Action}

It is easy to ascertain that a body which floats on the surface of a liquid is home to a downward-directed mechanical action. Let us assume that the volume of the body is B, and that, at equilibrium, the part of B which is immersed in the liquid is $\mathrm{v}$. We ideally assume that part $(\mathrm{B}-\mathrm{v})$ which is not immersed in the liquid is immersed in a vacuum.

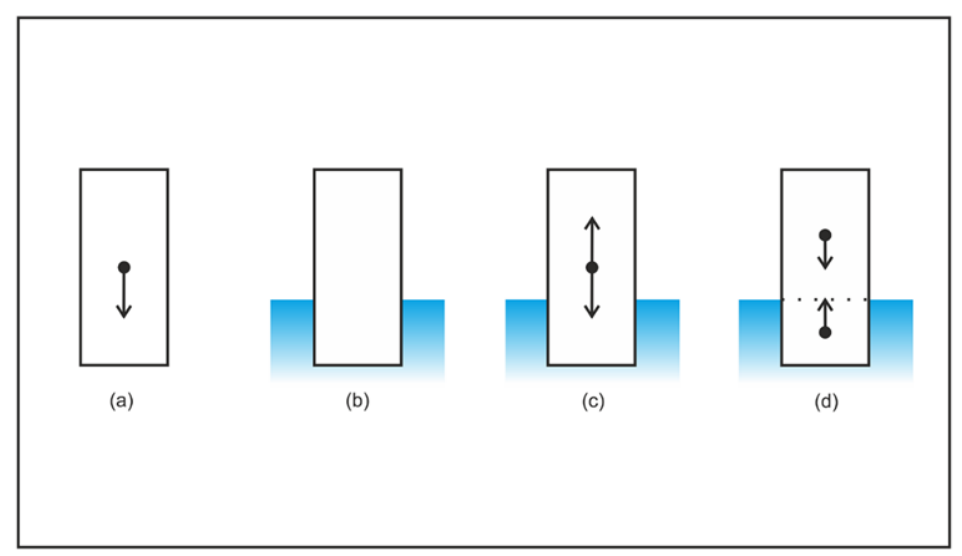

Figure 1. (a) A material volume immersed in a vacuum; (b) The material volume which floats on the surface of a liquid; (c) Current interpretation of the equilibrium of floating; (d) Alternative interpretation of the equilibrium of floating (From Cavazzini, 2019)

Let us accurately measure the part $\mathrm{v}$ of the volume of the body, or the part $(\mathrm{B}-\mathrm{v})$, and let us prepare two separate volumes of the same material which constitutes body $\mathrm{B}$, one volume exactly equivalent to part $\mathrm{v}$, and the other volume equivalent to part (B - v).

Then we let volume $\mathrm{v}$ to float on the surface of the liquid, and, at equilibrium, we let volume (B -v) to rest on the top of volume $\mathrm{v}$. Volume $\mathrm{v}$ gradually dips into the liquid until it is completely immersed, and, at equilibrium, no part of volume $(\mathrm{B}-\mathrm{v})$ is immersed in the liquid.

Since volume (B - v), when it is completely immersed in a vacuum, is home to a downward-directed mechanical 
action, this volume $(\mathrm{B}-\mathrm{v})$ is home to an equivalent downward-directed mechanical action also when we let it to rest on the top of volume $\mathrm{v}$ floating on the surface of the liquid, because volume $(\mathrm{B}-\mathrm{v})$, at equilibrium, is completely immersed in a vacuum. Therefore, since this equilibrium situation exactly reproduces the floating equilibrium of body B - it exactly reproduces the volume which is completely immersed in the liquid and the volume which is completely not immersed in the liquid - it is evident that the volume immersed in the liquid, $\mathrm{v}$, is home to an upward-directed mechanical action which balances the downward-directed mechanical action of which is home volume $(\mathrm{B}-\mathrm{v})$ immersed in a vacuum.

In both these situations of mechanical equilibrium (the mechanical equilibrium of volume $\mathrm{B}$, and the mechanical equilibrium of the two separate volumes $\mathrm{v}$ and $(\mathrm{B}-\mathrm{v})$ ), however, we do not know the intensity of the downward directed mechanical action. We know the intensity of the downward-directed action of which volume $(\mathrm{B}-\mathrm{v})$ is home, but we do not know whether volume $\mathrm{v}$ immersed in the liquid is also home to a downward-directed mechanical action or not. Consequently, we do not know the intensity of the upward-directed mechanical action of which volume $\mathrm{v}$ is home, because we do not know the 'composition' of this upward-directed mechanical action which balances the downward-directed action of volume $(\mathrm{B}-\mathrm{v})$.

\section{The Question of the Upward-Directed Mechanical Action of Which the Volume Which Is Immersed in the Liquid Is Home}

We do not know the 'composition' of the upward-directed action of volume $\mathrm{v}$ immersed in the liquid. By 'composition' we mean that we do not know whether

(a) the part of the volume of the body which is immersed in the liquid, v, when it is immersed in the liquid does not 'lose' its own weight, i.e., it is still home of the downward-directed mechanical action of which it is home when it is immersed in a vacuum

or whether

(b) the part of the volume of the body which is immersed in the liquid, when it is immersed in the liquid, 'loses' its own weight, i.e., it is no longer home of the downward-directed mechanical action of which it is home when it is immersed in a vacuum.

When a body is floating on the surface of a liquid, if the part of the body's volume which is immersed in the liquid 'loses' its own weight, the intensity of the upward-directed action of which the immersed volume is home must be equivalent to the intensity of the downward-directed action of which is home the part of the volume of the body which is not immersed in the liquid.

Instead, if the part of the volume of the body which is immersed in the liquid does not lose its own weight, the intensity of the upward-directed mechanical action of which this part of the volume of the body becomes home when it is immersed in the liquid is equivalent to the weight of the whole body. (We remember the reader that whenever we write the word 'weight' of a volume of matter, we mean the downward-directed mechanical action of which the volume of matter is home when it is immersed in a vacuum).

\section{The Two Hypotheses}

Evidently, the two hypotheses are both possible. In principle, however, they cannot both be true. Since the 'weight' of a volume of matter is one of the effects of the gravitational process, the question of what is the correct physical interpretation when a body is floating on the surface of a liquid provides significant information about the gravitational process. The current interpretation of floating of a body on the surface of a liquid - the 'Archimedes' Principle' (Archimedes, Halliday et al, 2001) - assumes hypothesis (a) as true (Cavazzini, 2018; 2019).

\section{The Formulas of the Two Hypotheses}

According to current view, all other conditions being equal, the 'weight' of a body is proportional to the 'mass' of the body, which is defined as the product between the volume of the body and the 'density' of the matter which constitutes the body. If we call $d, d_{\text {liq }}$, respectively, the density of the matter which constitutes the body and the density of the matter which constitutes the liquid, hypothesis (a) is:

$$
v d l i q=B d
$$

in which $v d_{l i q}$ represents the intensity of the upward-directed mechanical action of which is home volume $\mathrm{v}$, whereas hypothesis (b) is:

$$
-v\left(d-d_{\text {liq }}\right)=(B-v)(d-0)
$$

in which $-v\left(d-d_{l i q}\right)$ represents the intensity of the upward-directed mechanical action of which is home volume $\mathrm{v}$ 
(Cavazzini 2018; 2019).

As discussed in the previous sections, we ideally assume that the part of volume B which is not immersed in the liquid is immersed in a vacuum, and that the density of vacuum is exactly 0 , so that formulas (a) and (b) can be perfectly reduced one to each other from the quantitative viewpoint with no loss of precision.

\section{Mathematics Cannot Distinguish the Two Interpretations Despite They Are Very Different from the Physical Viewpoint}

It is important to note that formula (b) can be readily reduced to formula (a) from a mathematical point of view, by decomposing the first member in (b) to the algebraic sum of two addenda (according to current physical interpretation/definition of 'weight', the two addenda can be interpreted as two 'weights').

However, this decomposition is not possible from the physical viewpoint, because the first member in (b) represents a single mechanical action, which is proportional to the volume which is immersed in the liquid and to the difference in density between the matter which constitutes the volume and the matter which constitutes the liquid.

Therefore, mathematics, via our interpretation/definition of 'weight', gives rise to physical ambiguity. These two formulas can be reduced one to each other from the mathematical viewpoint, but they cannot be reduced one to each other from the physical viewpoint, because the two opposing mechanical actions which give rise to the mechanical equilibrium of the body which floats on the surface of the liquid have different intensity. In the case of formula (b), the intensity of the two opposing mechanical actions is lower than in the case of formula (a) (compare the lengths of the arrows in Fig 1 (c) and (d), which represent hypotheses (a) and (b), respectively).

The two formulas represent very different physical scenarios. In the case of hypothesis (a) (Fig. 1 (c)), the part of the volume of the body which is immersed in the liquid, $v$, is home to two mechanical actions, its own weight i.e., the downward-directed action of which this volume is home when it is immersed in a vacuum - and the so-called 'Archimedes' Force', directed in the opposite direction, the intensity of which is equal to the weight of a volume of liquid equivalent to the part of the volume of the body which is immersed in the liquid. In the case of hypothesis (b), instead, the volume which is immersed in the liquid is home to a single mechanical action directed upwards, the intensity of which is proportional to the volume immersed in the liquid and to the difference in density between the matter which constitutes the volume and the matter which constitutes the liquid (Fig. 1 (d)).

\section{How to Decide}

To establish what interpretation is the correct one, we have to be able to show that one of these two interpretations leads to contradiction. If hypothesis (a) leads to contradiction whereas hypothesis (b) does not lead to contradiction, (b) is the correct interpretation; vice-versa, (a) is the correct interpretation.

It is also possible that both of these hypotheses do not lead to any contradiction. In this case, they would be possible alternatives. Such a situation of indecisiveness, however, would show the limits of our interpretative approach. We have already shown that this occurs from the quantitative viewpoint, because formulas (a) and (b) can be reduced one to each other from the quantitative viewpoint without losing precision.

In order to prove the existence of a contradiction, we need to refer to facts. Facts cannot be questioned.

\section{The fact}

For a material volume which floats on the surface of a liquid, the only fact is the following:

\section{All other conditions being equal, at equilibrium, the quantitative ratio between the volume of the part of the body which is immersed in the liquid and the volume of the part of the body which is not immersed in the liquid is fixed.}

or, which is the same,

All other conditions being equal, at equilibrium, the quantitative ratio between the volume of the part of the body which is immersed in the liquid and the volume of the body is fixed. (This parameter is the 'immersed fraction' of the body).

In other words, when a body is floating on the surface of a liquid, at equilibrium, the ratio $\mathrm{R}$ between the volume of the part of the body which is immersed in the liquid and the volume of the part of the body which is not immersed in the liquid, and the immersed fraction F, all other conditions being equal, are independent of the volume of the body which floats. By 'conditions' we mean all the external conditions, and the density value of the matter which constitutes the body and the density value of the matter which constitutes the liquid. 
The relationship between $\mathrm{F}$ and $\mathrm{R}$ is:

$$
F=R /(1+R)
$$

The values of $\mathrm{R}$ and $\mathrm{F}$ characterize mechanical equilibrium. In particular - see Section 4. - ratio $\mathrm{R}$ gives the quantitative relationship between a volume which is (completely) immersed in the liquid and a volume which is (completely) not immersed in the liquid, at equilibrium. Values of $\mathrm{R}$ which are not appropriate indicate situations of mechanical disequilibrium.

In the following, we assume that $d_{l i q}=3 d$, so that $\mathrm{v}=\mathrm{B} / 3$ (see equivalences (a) and (b)), ratio $\mathrm{R}$ is $1 / 2(\mathrm{v}=\mathrm{B} / 3$ and $(B-v)=2(B / 3))$, and immersed fraction $F$ is $1 / 3$.

\section{An Ideal Experiment}

Let us consider volume $\mathrm{B}$ which floats on the surface of the liquid. At equilibrium, a part $\mathrm{v}$ of this volume is immersed in the liquid. We assume that the part $(\mathrm{B}-\mathrm{v})$ of volume $\mathrm{B}$ is immersed in a vacuum. We call this mechanical equilibrium 'equilibrium (1)'.

Let us now rest a volume $\mathrm{A}$ on the top of volume $\mathrm{B}$. Let volume $\mathrm{A}$ constituted by the same matter which constitutes volume B, and let A = 2B (see Cavazzini, 2018; 2019). In this case, the part (B - v) of volume B, initially not immersed in the liquid, is progressively immersed in the liquid. At equilibrium, volume B is completely immersed, whereas no part of volume A is immersed in the liquid. Let we call this mechanical equilibrium 'equilibrium (2)'.

\section{Current Interpretation Leads to Contradict an Observed Fact}

Let us assume that hypothesis (a) is correct. Accordingly, in the case of volume B which floats alone on the surface of the liquid, the part $\mathrm{v}$ of this volume which is immersed in the liquid, when it is immersed in the liquid, does not lose its own weight. Thus, in equilibrium (1), part $\mathrm{v}$ is home to an upward-directed mechanical action which balances the weight of the whole volume $B$.

Let us now rest volume $A$ on the top of volume $B$. Volume $(B-v)$ is progressively immersed in the liquid, and from equilibrium (1) the situation changes to equilibrium (2). According to hypothesis (a), volume (B - v) does not lose its own weight when it is immersed in the liquid. Therefore, in equilibrium (2), the upward-directed mechanical action of volume $\mathrm{v}$ immersed in the liquid still balances the weight of volume $\mathrm{B}$, i.e., equilibrium (1) is unaltered passing from equilibrium (1) to equilibrium (2).

We can say that equilibrium (1) is kept unaltered 'within' equilibrium (2), because volume $\mathrm{v}$, which is immersed in the liquid in equilibrium (1), is immersed in the liquid also in equilibrium (2), so that we are sure that the mechanical action of which volume $\mathrm{v}$ is home does not change. Since, according to hypothesis (a), equilibrium (1) is the mechanical equilibrium between the upward-directed mechanical action of which is home volume $\mathrm{v}$ immersed in the liquid and the downward-directed mechanical action of which is home volume B (the weight of volume $\mathrm{B}$ ), and volume (B - v) does not lose its own weight when it is immersed in the liquid, equilibrium (1) is maintained within equilibrium (2). This means that the upward-directed mechanical action of which is home volume $\mathrm{v}$ immersed in the liquid does not concur in generating equilibrium (2). This means also that a mechanical equilibrium must be added to equilibrium (1) to obtain equilibrium (2), because the addition of a mechanical disequilibrium to a mechanical equilibrium inevitably gives rise to a mechanical disequilibrium.

Therefore, if hypothesis (a) is correct, it is the upward-directed action of which is home volume (B - v) immersed in the liquid which balances the mechanical action of which is home volume A not immersed in the liquid. In other words, the volume (completely) not immersed in the liquid involved in the mechanical equilibrium which must be added to equilibrium (1) to give rise to equilibrium (2) is A, and the volume (completely) immersed in the liquid involved in the mechanical equilibrium which must be added to equilibrium (1) to give equilibrium (2) is $(\mathrm{B}-\mathrm{v})$.

We can check if this is a condition of mechanical equilibrium by calculating the value of ratio $R$, which is the quantitative ratio at equilibrium between a volume which is (completely) immersed in the liquid and a volume which is (completely) not immersed in the liquid (see Section 4.). Ratio $R$ is $(B-B / 3) / 2 B=1 / 3$, so indicating a condition of mechanical disequilibrium. Therefore, hypothesis (a) leads to contradict an observed fact, i.e., the fact that equilibrium (2) is a condition of mechanical equilibrium, because it is not possible to obtain a mechanical equilibrium (equilibrium (2)) by adding to a mechanical equilibrium (equilibrium (1)) a mechanical disequilibrium.

\section{Equilibrium (1) Turns into a Disequilibrium}

We infer that when we let volume A to rest on the top of volume B, and volume (B - v) is progressively 
immersed in the liquid, equilibrium (1) is altered, turning into a mechanical disequilibrium. It is this mechanical disequilibrium which gives rise to equilibrium (2) by addition with the mechanical disequilibrium which is given by the upward-directed mechanical action of which is home volume $(\mathrm{B}-\mathrm{v})$ immersed in the liquid and the downward-directed action of which is home volume A not immersed in the liquid.

In other words, equilibrium (2) is the result of the concurrent action of the upward-directed action of volume $\mathrm{v}$ immersed in the liquid, and of the upward-directed action of volume (B - v) immersed in the liquid, which, together, balance the downward-directed mechanical action of volume A immersed in a vacuum. This, however, can occur only if volume $(B-v)$, when it is immersed in the liquid, loses its own weight, i.e., it can occur only if hypothesis (b) is true. This means that also volume $\mathrm{v}$, when it is immersed in the liquid, loses its own weight. The error is in interpreting equilibrium (1) as currently, i.e., according to hypothesis/formula (a).

\section{The Correct Interpretation}

Since after volume A has been let to rest on the top of volume B a condition of mechanical equilibrium is reached in which no part of volume $\mathrm{A}$ is immersed in the liquid, we deduce that the upward-directed action of volume $\mathrm{v}$ immersed in the liquid concurs with the upward-directed action of volume $(\mathrm{B}-\mathrm{v})$ immersed in the liquid in balancing the downward-directed action of volume A not immersed in the liquid (immersed in a vacuum). This means that volume (B-v), when it is immersed in the liquid, loses its own weight, because, if it were not so, equilibrium (1) would be kept unaltered within equilibrium (2), and in this case, the value of the ratio between volume (B - v) completely immersed in the liquid and volume A completely not immersed in the liquid would have been the equilibrium value. Thus, hypothesis (a) - Fig. 1(c) - cannot explain mechanical equilibrium (2), and it must be rejected as false.

Interpretation (b) - Fig. 1(d) - is the correct interpretation of the mechanical equilibrium of a body which floats on the surface of a liquid. Accordingly, we write equilibrium (1) of volume B which floats alone on the surface of the liquid as::

$$
-v\left(d-d_{\text {liq }}\right)=(B-v)(d-0)
$$

which must be read as follows:

The upward-directed mechanical action of which is home volume $v$ immersed in the liquid, which is proportional to the immersed volume, $\mathrm{v}$, and to the difference in density between the matter which constitutes the volume and the matter which constitutes the liquid, $\left(d-d_{\text {liq }}\right)$, balances the downward-directed mechanical action of which is home volume (B - v) immersed in a vacuum, action which is proportional to the volume immersed in a vacuum, $(B-v)$, and to the difference in density between the matter which constitutes the volume immersed in a vacuum and the 'matter' that constitutes the vacuum.

\section{Consequences and Conclusions}

According to the line of reasoning here illustrated, interpretation (a) leads to contradict observed facts, whereas interpretation (b) does not lead to any contradiction. Therefore, interpretation (a) is false, whereas interpretation (b) - Fig. 1(d) - is the true interpretation:

A volume of matter, when it is immersed in a fluid, 'loses' its own 'weight', i.e., it is no longer home of the downward-directed mechanical action of which it is home when it is immersed in a vacuum.

In other words, a material volume $V$ immersed in a fluid does not simultaneously host two opposite mechanical actions (the 'weight' of the volume, as if it were immersed in a vacuum, $V d$, directed downwards, and an upward-directed action the intensity of which is equal to the 'weight' of a volume of fluid equivalent to immersed volume $V, V d_{\text {liq }}$, the so-called 'Archimedes' Force') which 'compose' to give a mechanical action directed upwards, or downwards, or to give no net mechanical action. Instead, volume $V$ is home to a single mechanical action the intensity of which is proportional to the volume immersed in the fluid, and to the difference in density between the matter which constitutes volume $V$ and the matter which constitutes the fluid, $V\left(d-d_{\text {fluid }}\right)$.

When body B is floating on the surface of the liquid, at equilibrium, the upward-directed mechanical action of which is home the part $\mathrm{v}$ which is immersed in the liquid is not the algebraic sum of two mechanical actions (two 'weights', according to current definition/interpretation of 'weight'):

$$
-v d+v d_{l i q}
$$

but a single mechanical action, proportional to the volume immersed in the liquid and to the difference in density between the matter which constitutes the volume and the matter which constitutes the liquid:

$$
-v\left(d-d_{l i q}\right)
$$


Once again it is clear that these two interpretations cannot be distinguished from the mathematical viewpoint, in spite of the important difference in physical meaning, and the evident ambiguity is increased by the current definition/interpretation of 'weight', because (4) can be interpreted as the algebraic sum of two weights.

Comparing interpretations (a) and (b) shows that interpretation (b) meets William of Ockham's methodological 'rule' called 'Ockham's razor'. Volume v immersed in the liquid is home to only one mechanical action and not home to two mechanical actions (Entia non sunt multiplicanda praeter necessitate). As we have discussed above, this mechanical action (eq. (4)) could be interpreted as the algebraic sum of two actions as proposed in interpretation (a) - two 'weights', according to the current definition/interpretation of 'weight' (eq. (3)) - but, however, it is not the sum of such two actions. This leads us to radically change the physical interpretation of the phenomenon.

Since a material volume immersed in a fluid is home to a single mechanical action, and since we observe that in some cases the same material volume, when immersed in some fluids, is moved in one direction, whereas when it is immersed in other different fluids it is moved in the opposite direction, we infer that

The direction of the mechanical action of which is home a material volume immersed in a fluid is related to the sign of the difference in density between the matter which constitutes the volume and the matter which constitutes the fluid. The direction of the mechanical action reverses as the sign of the difference in density changes from positive to negative, or vice-versa.

Moreover, since the mechanical action is proportional to the difference in density between the matter which constitutes the body and the matter which constitutes the fluid:

The mechanical action is $\mathbf{0}$ if the difference in density is $\mathbf{0}$, i.e., the mechanical action is $\mathbf{0}$ if equilibrium in density exists between the matter which constitutes the body and the matter which constitutes the fluid.

And, in other words:

The mechanical action 'ceases' if equilibrium in density is reached between the matter which constitutes the body and the matter which constitutes the fluid.

This means that:

The main cause of this mechanical action is related to the disequilibrium in density between the matter which constitutes the body and the matter which constitutes the fluid.

And, since the matter of the fluid is the direct surroundings of the matter which constitutes the body, and vice-versa:

The main cause at the origin of this mechanical action is a physical disequilibrium between matter which is in contact.

Since this mechanical action ceases when equilibrium in density is reached between the matter which constitutes the body and the matter which constitutes the fluid, i.e., the mechanical action ceases when equilibrium is reached between the matter of the body and the matter of the fluid in an intensive property of the matter we call 'density', we realize that:

This mechanical action is the manifestation of a physical process which shows characteristics which are typical of the spontaneous processes.

Finally, what this mechanical action "is"? Since a hypothesis about the 'weight' - i.e., about the gravitational mechanical action - which is currently believed to be true is instead false and it should be abandoned, we propose to call this mechanical action the 'gravitational action of which a body is home when it is immersed in a certain fluid'. In other words, we propose to call this mechanical action 'weight of the body in that fluid', ceteris paribus, i.e., all other conditions being equal (Cavazzini, 2018; 2019).

Therefore, if we change our interpretative framework of this physical process, gravitation can be added to the 'field' of the 'spontaneous processes' (Cavazzini, 2020). Only spontaneous processes are possible in the Universe, and gravitation is evidently a process which occurs spontaneously. As in the case of any other spontaneous process, the gravitational process takes origin from a disequilibrium in the value of an 'intensive' 'property' of the matter, the disequilibrium in 'density' between the matter which constitutes the body and the matter which constitutes the fluid, This disequilibrium is the basis of this spontaneous physical process, and the aim of the process, as in the case of any other spontaneous process, is reducing/eliminating the disequilibrium (Cavazzini, 2019; 2020).

Acknowledgements 
This work was financially supported by C.N.R. Istituto di Geoscienze e Georisorse.

\section{Conflict of interests}

The authors declare that there is no conflict of interests regarding the publication of this paper.

\section{References}

Archimedes of Syracuse. On floating bodies, Book I (in Italian, in Opere di Archimede; Torino, UTET, 1974).

Bierman, J., \& Kincanon, E. (2003). Reconsidering Archimedes' Principle. The Physics Teacher, 41, 340-344. https://doi.org/10.1119/1.1607804

Brown, R. (1999). Weight - don't use the word at all. The Physics Teacher, 37, 241. https://doi.org/10.1119/1.880241

Cavazzini, G. (2018). A New Physical Interpretation of Archimedes' Principle. Journal of Applied Mathematics and Physics, 6(1), 215-233. https://doi.org/10.4236/jamp.2018.61020

Cavazzini, G. (2019). Archimedes' Principle and the Concept of Gravitation. Applied Physics Research, 11(6), 9-18. https://doi.org/10.5539/apr.v11n6p9

Cavazzini, G. (2020). Archimedes' Principle and Gravitation as a Spontaneous Process. Applied Physics Research, 12(1), 1-10. https://doi.org/10.5539/apr.v12n3p1

Graf, E. H. (2004). Just what did Archimedes say about buoyancy? The Physics Teacher, 42, 296-299. https://doi.org/10.1119/1.1737965

Halliday, D., Resnick, R., \& Walker, J. (2001). Fundamentals of Physics (6th ed.). New York, Wiley.

Kubrick, S. (Director). (1980). The Shining [Motion picture]. United States: Warner Brothers.

Leroy, B. (1985). Archimedes' Principle: A simple derivation. European Journal of Physics, 6, 56. https://doi.org/10.1088/0143-0807/6/1/107

Sears, F. W. (1963). Weight and weightlessness. The Physics Teacher, 1, 20-23. https://doi.org/10.1119/1.2350557

Taibu, R. (2015). Terms vs, concepts - the case of weight. The Physics Teacher, 55, 34-35. https://doi.org/10.1119/1.4972495

Taibu, R., Rudge, D., \& Schuster, D. (2015). Textbooks presentations of weight: Conceptual difficulties and language ambiguities. Physical Review Education Research, 11, 1-20. https://doi.org/10.1103/PhysRevSTPER.11.010117

\section{Copyrights}

Copyright for this article is retained by the author(s), with first publication rights granted to the journal.

This is an open-access article distributed under the terms and conditions of the Creative Commons Attribution license (http://creativecommons.org/licenses/by/4.0/). 